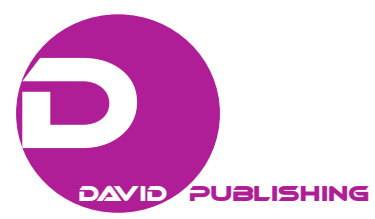

\title{
Holistic Approach and Development
}

\author{
Taysir Nashif \\ United Nations
}

\begin{abstract}
According to the holistic approach, factors relevant for the promotion of a desire of change should be accommodated in a synchronized manner. Need to implement this approach is the topic of this paper. Put differently, the topic of research has to do with desired change through implementation of holistic approach.

Keywords: Change, Development, Holistic Approach, Factor, Effects Inadequacy Information
\end{abstract}

\section{Introduction}

In spite of the efforts which developing countries in Asia, Africa, Latin America and other areas have made and continue to make in order to achieve desired change to attain socio-political and economic development, no satisfactory results have been made.

\section{Research Topic}

In space, including the Earth, there are factors which are relevant for the promotion of desired change. These factors need to be accommodated in a synchronized manner. What makes accommodation difficult to achieve is the existence of hidden and unknown factors in the cosmos, beyond the known world. Holistic approach can contribute to the achievement of objectives.

\section{Method}

Factors in the universe have mutual, uneven effects. Major factors have a greater presence and influence. The method of study is empirical, quantitative and analytical. Historical, political and economic considerationshave their influence. An assumption is made that the universal system is dynamic with varyingdegrees of ethical, political and physical effects. The treatment relies on sources, mainly socio-cultural and economic statistical data available at the United Nations Statistical Library and the United Nations Development Program, and covering the developing countries for the last thirty years.

\section{Dynamic Factors and Holistic Approach}

Dynamic factors, which are abundant in our universe, are influential factors which keep interacting with other influential factors. In order to be able to achieve desired change, a holistic approach needs to be adopted. According to this approach, factors relevant for the promotion of the desired change, such as spread of learning and establishment of infrastructure, should be accommodated and utilized in a synchronized manner. Otherwise,

Taysir Nashif,.Ph.D., political science, SUNY-Binghamton. 
existing cultural, economic, psychological and political (social, in the broader sense) factors, such as a very high birth rate and an inadequate medical system, which are unfriendly to the factors relevant for the process of the promotion of the desired change, would vitiate or undo the process of such change. The method of study is empirical, quantitative, analytical and historical.

\section{Holistic Approach and Development}

Even though the universe is regarded as naturally dynamic, it follows certain rules of order. This also applies to the Earth. There are so many factors in the universe. What is meant by a factor is that entity which creates influence and, or is, faced with influence. Dynamic factors acquire their balance by the dynamic relations and inter-relations among the factors. These factors are either stronger one from the others; they play negative or passive role vis-a-vis the other factors, their fields of activity may be different one from the other.

It is obvious that in all human societies, cultural, economic, psychological and political activity, learning, teaching, technology, expertise, authority, information, law, rule and others, exist. These factors either have varying degrees of good effect, negative effect, or neutral effect, in terms of their intended or unintended effects, depending on the prevailing ethical environment and on their relevance or lack of it for the other existing factors. Major factors, such as culture, ethics, law and rule, exert more significant influence. The ethical or value orientation of a context, the cultural and economic vision of the people, the human authority do constitute a major source of influence in a given context.

These major factors are the role players that determine the nature of the goal within a context. Within a certain or un-certain context, the role of a major factor, such as economics or value orientation, is a result of the other factors' roles when interacting with one another and with the given major factor. According to the holistic approach, human decision making bodies should be sensitive to the changing structure of the major factors and to the other factors. Advancement, development, progress require that attention given to the major factors is proportional to the condition of the major factor.

Moreover, in order for factors of development to be effective, the counteracting factors causing backwardness need to be coped with. Whatever achievements the factors of development can achieve in this direction would be rendered insignificant or vitiated by the factors that work in the direction of backwardness. It bears repetition that, to cope with this condition, a holistic approach needs to be adopted in order to take measures conducive to the achievement of development.

The dynamic character of the factors also makes necessary that adoption of a holistic approach. Because of varying social, cultural, economic and political backgrounds, a factor may have different meanings and definitions. Whereas, according to some Western scholars, "politics" has to do with influence and the influential, and the "influential are those who get the most of what there is to get," (Lasswell, 1971), "politics," according to a number of scholars in the Middle East, means to lead people for the people's interests. (Anis, \& others, 1972). Multitude of definitions of one factor tends to create room for different people to attach different meanings to one factor, depending on time and location. The dynamic character of a factor, depending on the social-political-economic background, has its implications for the treatment of development. Thus, the relatively few people ancient Athens made the democratic practice participatory, whereas, with a population amounting to tens of millions in the UK, the representative democracy was seen to be unsuitable. The character 
of factors may have bearing on the ordering or re-ordering of the components of the development process. It is through the adoption of a holistic approach that the implications of such dynamic character are accommodated.

Given the dynamic character of factors and of social climate in which a factor emerges and is manifested, measuring of the effect of social factors undergoes change. The effect of the social factors is the product of the interaction of all the factors in a conditioning social context. The description of a certain effect of such factors as developmental, status quo, or backward-oriented is the result of the interaction of such factors at a certain time and place. As this configuration of factors is dynamic, effect which under certain conditioning circumstances may be categorized as status quo or stagnating may under different circumstances be described as backward-oriented or developmental. Science, which under a certain governing regime which utilizes it for the promotion of public interests, deserves to be labeled as developmental, may, under a governing regime which uses it in ways that weaken the survivability of natural creatures, deserves to be described as backward-oriented.

\section{Conclusion}

Earthly and unearthly factors are influenced by, and do influence, other factors. No evidence does exist that attention given by human authorities to factors was adequate to produce development on the international level. To achieve holistic approach, relevant healthy approach, requiring organization, coordination and study, needs to be undertaken.

\section{References}

Anis, I., \& others (1972).Al-Mu'jam Al-Wasit. Cairo: Dar Al-Ma’arif. Lasswell, H. D. (1971). Politics: Who gets what, when, how. New York: Meridian.

Nashif, T. (2011). Social justice and intellectual suppression. Bloomington: Indiana. 